\title{
Accumulation Ratio AUC Over Dosing Interval Normalized by Dose
}

National Cancer Institute

\section{Source}

National Cancer Institute. Accumulation Ratio AUC Over Dosing Interval Normalized by

Dose. NCl Thesaurus. Code C132435.

The area under the curve (AUCtau) at steady state divided by the area under the curve (AUCtau) over the initial dosing interval, each divided by the associated dose. 